\title{
Analyse statistique des pluies journalières extrêmes à partir d'un seuil dans le Bénin subéquatorial
}

\section{HOUNVOU S. F. ${ }^{1}$, GUEDJE F. K. ${ }^{2}$, KOUGBEAGBEDE H. ${ }^{3}$, ADECHINAN J. ${ }^{4}$, HOUNGNINOU E. 5}

\author{
${ }^{1}$ Laboratoire de physique de l'atmosphère,FAST/UAC, ferdilu25@gmail.com \\ ${ }^{2}$ Laboratoire de physique de l'atmosphère, FAST/UAC, guedjefranco@yahoo.com \\ ${ }^{3}$ Laboratoire de physique de l'atmosphère,FAST/UAC, hilars83@gmail.com \\ ${ }^{4}$ Laboratoire de physique de l'atmosphère,FAST/UNSTIM, adjos85@gmail.com \\ ${ }^{5}$ Laboratoire de physique de l'atmosphère,FAST/UAC, houngnb@gmail.com
}

\section{INFOS SUR L'A R T I C L E}

Historique de l'article:

Reçu le : 31 juillet 2019

Reçu en format revisé le : 23 décembre 2019

Accepté le : 31 décembre 2019

\begin{abstract}
Mots-Clés:Variabilité climatique, analyse statistique, pluies extrêmes, seuil, loi de Pareto, Bénin subéquatorial.
\end{abstract}

Keywords :Climate variability, statistical analysis, threshold, Pareto law, daily rainfall, Benin subequatorial.

\begin{abstract}
Résumé : La forte variabilité du climat de l'Afrique de l'ouest à des impacts socio-économiques sévères sur la vie des habitants. Il urge de mieux comprendre les mécanismes physiques de celle-ci pour améliorer la prédétermination et la prévision des inondations et des sécheresses dans cette région du monde. L'analyse des pluies extrêmes supérieures à un seuil, est faite à cet effet dans le Bénin subéquatorial. Les données de pluies journalières exploitées sont recueillies sur dix-sept (17) stations au sud du Bénin. Les résultats montrent des seuils variants entre $17,4973 \mathrm{~mm}$ et $37,9033 \mathrm{~mm}$ d'une part et une croissance du nombre annuel des hauteurs de pluie supérieures à un seuil après la reprise de la pluie des années 80 ou du début des années 90 sur les différentes stations d'étude. Avec un paramètre de forme significativement négatif, la loi généralisée de Pareto permet de bien ajuster les pluies journalières supérieures à un seuil.
\end{abstract}

\begin{abstract}
West Africa's high climate variability has severe socioeconomic impacts on people's lives. There is a need to better understand its physical mechanisms to improve the prediction and prediction of floods and droughts in this region of the world. The analysis of extreme rainfall above a threshold is made for this purpose in subequatorial Benin. Daily rainfall data are collected seventeen (17) stations in southern Benin. The results show thresholds ranging between $17.4973 \mathrm{~mm}$ and $37.9033 \mathrm{~mm}$ on the one hand and a growth in the annual number of rainfall heights greater than a threshold after the resumption of rain in the 1980s or early 1990s on the different study stations. With a significantly negative shape parameter, the Pareto generalized law allows to adjust well the daily rains superior to only one.
\end{abstract}

\section{INTRODUCTION}

L'Afrique de l'Ouest est l'une des régions du monde qui subissent de graves problèmes de crue et d'inondation, tant en milieu urbain que rural à cause de son climat caractérisé par une forte variabilité pluviométrique (Soro et al., 2016). Les conséquences sont parfois dramatiques à cause de la forte vulnérabilité de sa population aux extrêmes climatiques (Tschakert P., 2007). La spécificité des différentes mesures de prévention et de protection nécessite de bien connaître l'aléa (Panthou, 2013).

Le Bénin comme la plupart des pays de cette région $\mathrm{du}$ monde, a connu plusieurs pluies de fortes intensités qui pourraient être l'un des éléments majeurs des inondations de ces dernières années dans les différentes villes du pays. C'est le cas par exemple des inondations de 2010, qui ont causé surtout dans le sud Bénin, des dégâts importants sur les biens et services (rapport d'évaluation des besoins post catastrophe au Bénin, 2011). Il convient donc de faire une minutieuse analyse de la pluviométrie à travers la variabilité des pluies extremes.

L'approche statistique fréquemment utilisée est l'analyse des valeurs maximales annuelles de la pluie. Plusieurs auteurs (Koutsoyiannis et Baloutsos, 2000 ; Gellens, 2002 ; Sahani M. et al., 2012) ont utilisé cette approche surtout à cause de la simplicité de sa mise en œuvre à travers la loi de Gumbel afin de quantifier le 
risque associé aux pluies extrêmes. Cependant la seule valeur maximale annuelle retenue peut conduire au rejet de beaucoup d'autres évènements extrêmes. Ce qui peut restreindre l'échantillonnage des données de pluie pour l'analyse (Coles, 2001, Soro et al., 2016). La méthode des valeurs supérieures à un seuil permet de résoudre ce problème.

Encore appelée méthode des excès, cette méthode permet de fournir une caractérisation asymptotique de la distribution de dépassements de seuil (Pickands, 1975 ; Coles, 2001). Elle est particulièrement efficace pour extraire des informations concernant les véritables événements extrêmes (Acero et al., 2011 ; Saidi et al., 2013 ; Soro et al, 2016). Les dépassements de seuil extraits (de pluie ou tout autre variable hydrométéorologique) d'un échantillon peuvent être modélisés par les lois de Pareto généralisées dont la loi exponentielle est un cas particulier (Beirlant et al., 2004 ; J.N. Bacro et A. Chaouche, 2006). De nombreux auteurs ont utilisé cette approche pour estimer les valeurs

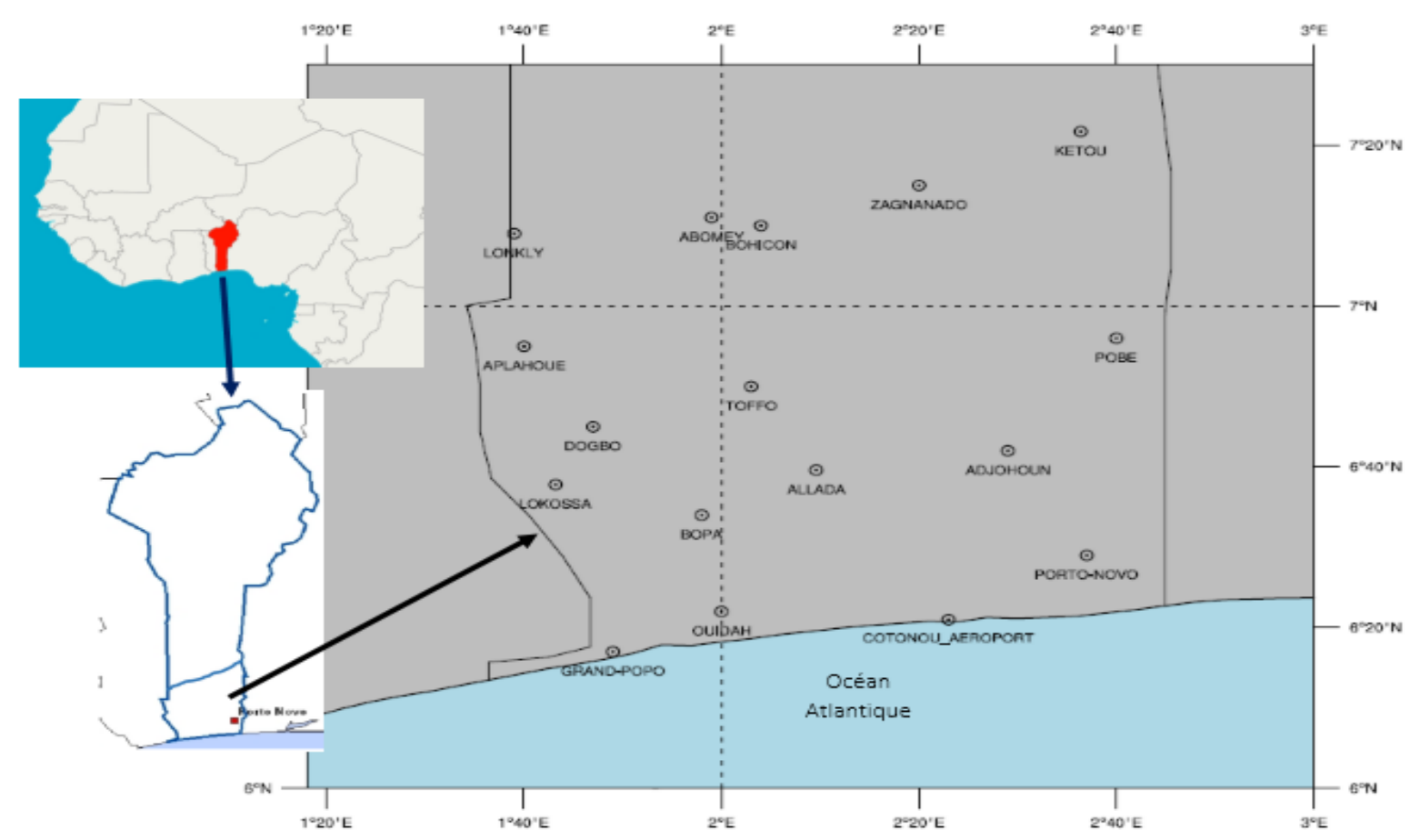

Fig.1. Situation géographique de la zone d'étude.

Les données utilisées dans cette étude sont des séries chronologiques de précipitations journalières recueillies sur 17 stations (voir figure 1b) de Météo Bénin. Deux stations (02) sont synoptiques (Cotonou et Bohicon) et le reste pluviométrique ou agro-climatique. Les hauteurs de pluies journalières sont recueillies entre 1980 et 2007 pour quatre (04) stations et de 1960 et 2012 sur les treize (13) autres. Les données manquantes n'excèdent pas 5\% dans les séries.

\section{I.2. Méthodes}

La fonction moyenne des excès est utilisée dans cette étude pour déterminer le seuil de pluie sur les différentes extrêmes de température et de pluie (M. Nogaj et al.,2006 ; Coelho et al., 2008 ; J.J. Egozcue et C. Ramis, 2001 ; S. Beguería et M. Vicente-Serrano, 2006 ; L. Tomassini et D. Jacob, 2009 ; M. RE et V.R. Barros, 2009 ; F. Serinaldi ; C.G. Kilsby, 2014 cités par Soro et al., 2016).

Dans le présent travail, les hauteurs de pluies journalières à partir d'un seuil sont analysées et modélisées par la distribution de Pareto généralisée (GDP) et la loi exponentielle qui est son cas particulier.

\section{DONNEES ET METHODES}

\section{I.1. Données}

S'étendant de Cotonou (au sud), sur la côte atlantique, jusqu'à la latitude de Bohicon (au nord) sur le socle Dahoméen, le Bénin subéquatorial est situé entre $1^{\circ} 37^{\prime}$ et $2^{\circ} 44^{\prime}$ E et $6^{\circ} 14^{\prime}$ et $7^{\circ} 22^{\prime} \mathrm{N}$. Il couvre une superficie de $\left(14111,0811 \mathrm{Km}^{2}\right)$. Le sud du Bénin est soumis à un régime pluviométrique bimodal. La grande saison s'étale de mars en juillet et la petite de septembre à novembre (Boko, 1992 ; Adewi et al., 2010).

stations. Elle a permis de définir l'intervalle (zone de linéarité) dans lequel se trouve le seuil adéquat. Cette approche proposée par Coles (2001) repose sur la stabilité des estimateurs des paramètres du modèle statistique sur une gamme de seuils. La gamme de seuils est celle proposée par la fonction moyenne des excès. Le seuil sur chaque station est estimé par la moyenne des différentes hauteurs de pluie de la gamme de seuils déterminée. Les détails concernant ces procédures de sélection du seuil d'estimation des évènements extrêmes sont donnés par Coles (2001). 
Tableau 1 : Les coordonnées géographiques des stations d'étude

\begin{tabular}{|l|l|l|l|l|}
\hline Stations & $\begin{array}{l}\text { Longitude } \\
\left({ }^{\circ} \text { Est }\right)\end{array}$ & $\begin{array}{l}\text { Latitude } \\
\left({ }^{\circ} \text { Nord }\right)\end{array}$ & $\begin{array}{l}\text { Altitude } \\
(\mathbf{m})\end{array}$ & $\begin{array}{l}\text { Période des } \\
\text { données }\end{array}$ \\
\hline Abomey & 1,98 & 7,18 & 260 & $1960-2012$ \\
\hline Adjohoun & 2,48 & 6,70 & 60 & $1960-2012$ \\
\hline Bopa & 2,44 & 6,38 & 7 & $1960-2012$ \\
\hline Bohicon & 2,07 & 7,17 & 166 & $1960-2012$ \\
\hline Cotonou & 2,38 & 6,35 & 4 & $1960-2012$ \\
\hline $\begin{array}{l}\text { Grand- } \\
\text { Popo }\end{array}$ & 1,82 & 6,28 & 5 & $1960-2012$ \\
\hline Kétou & 2,60 & 7,35 & 118 & $1960-2012$ \\
\hline Lonkly & 1,69 & 7,15 & 110 & $1960-2012$ \\
\hline Ouidah & 2,08 & 6,37 & 10 & $1960-2012$ \\
\hline Pobè & 2,67 & 6,98 & 136 & $1960-2012$ \\
\hline $\begin{array}{l}\text { Porto- } \\
\text { Novo }\end{array}$ & 2,62 & 6,48 & 20 & $1960-2012$ \\
\hline Toffo & 2,08 & 6,85 & 37 & $1960-2012$ \\
\hline Zagnanado & 2,33 & 7,25 & 102 & $1960-2012$ \\
\hline Aplahoué & 1,67 & 6,92 & 153 & $1980-2007$ \\
\hline Allada & 2,16 & 6,66 & 46 & $1980-2007$ \\
\hline Lokossa & 1,72 & 6,63 & 31 & $1980-2007$ \\
\hline Dogbo & 1,78 & 6,8 & 56 & $1980-2007$ \\
\hline
\end{tabular}

La variabilité interannuelle du nombre annuel des pluies supérieures à un seuil est analysée sur chaque station. L'indice pluviométrique normalisé (Lawin et al., 2012) est déterminé sur les périodes de 1960-2012 d'une part et de 1980-2007 d'autre part, par : $I(i)=(P(i)-P)$ $/ \sigma$. Avec $P(i)$, le cumul annuel du nombre de jours de précipitation supérieure au seuil sur chacune des stations de la zone d'étude pour l'année i. P et $\sigma$ correspondent respectivement à la moyenne et à l'écart type de la série. La moyenne mobile a été utilisée pour rechercher la tendance à la hausse ou à la baisse dans la série.

Pour apprécier la qualité des séries à être indépendantes et identiquement distribuées, les tests de rupture de Pettitt et Buishand (Langet al. 2003) et celui de stationnarité de Kendall (Aka et al., 1996; Ague et Afouda, 2015) ont été appliqués aux hauteurs de pluie supérieures à un seuil sur chaque station pour vérifier les éventuels changements météorologiques (Lang et al., 2003). Les tests de corrélation de Pearson (Johnson et al., 1994) et d'homogénéité de Wilcoxon (Siegel, 1956) sont aussi appliqués.

Les pluies journalières supérieures à un seuil, sont ajustées par l'une des distributions de la loi de PARETO généralisée.

$$
F(x)=\left\{\begin{array}{l}
1-\left[1-\frac{\mathrm{k}(\mathrm{x}-\mathrm{u})}{\alpha}\right]^{\frac{1}{\mathrm{k}}} \text { pour } \mathrm{k} \neq 0\left(\begin{array}{c}
\text { loi de Pareto } \\
\text { généralisée }
\end{array}\right) \\
1-\exp \left[\frac{-\mathrm{x}-\mathrm{u}}{\alpha}\right] \text { pourk }=0 \text { (loi exponentielle) }
\end{array}\right.
$$

où $\mathrm{u}$, paramètre de position $(-\infty<\mathrm{u}<+\infty)$, représente le seuil d'estimation, $\alpha$ est le paramètre d'échelle $(\alpha>0)$ et $\mathrm{k}$ le paramètre de forme $(-\infty<\mathrm{k}<+\infty)$. Le domaine des valeurs possibles de la distribution de Pareto généralisée est donné par $\mathrm{u}<\mathrm{x}<\infty$ pour $\mathrm{k} \leq 0$ et par $\mathrm{u}<\mathrm{x}<\mathrm{u}+\alpha / \mathrm{k}$ pour $\mathrm{k} \geq 0$.

Pour faciliter les interprétations dans les différentes applications, en hydrologie par exemple, les quantiles élevés sont exprimés en fonction de période de retour plutôt qu'en termes de probabilité et appelés niveaux de retour. Une période de retour est le nombre moyen d'année entre un évènement passé et un autre de même ampleur ou hauteur. Il existe une relation simple entre la probabilité d'occurrence d'un événement correspondant au $\mathrm{p}$ - quantile et sa période de retour $\mathrm{T}(\mathrm{T} \geq 2: \mathrm{p}=1$ $-1 / T$ ou $T=1 /(1-p)$. Selon la classification internationale des événements extrêmes proposée par l'Institut Royal de la Météorologie de Belgique (Sene et Ozer, 2002 ; Sahani et al., 2012), une précipitation est qualifiée d' " anormale », de " très anormale », d' " exceptionnelle » ou de « très exceptionnelle » respectivement pour au moins une période de retour de 6 ans, 10 ans, 30 ans, et 100 ans. Des quantiles de pluies maximales journalières correspondant aux périodes de retour de 6 ans, 10 ans, 30 ans et 100 ans (avec un intervalle de confiance de 95\%) sont calculés sur les différentes stations.

Les critères d'information Bayésien (BIC) et d'Akaike (AIC) sont utilisés pour dégager la loi de la famille qui quantifie au mieux les valeurs de pluie supérieures à un seuil dans le Bénin subéquatorial.

\section{RESULTATS ET DISCUSSION}

\section{II.1. Estimation du seuil des pluies journalières extrêmes}

Les figures $2 \mathrm{a}$ et $2 \mathrm{~b}$ présentent la fonction moyenne des excès des pluies journalières à la station Lokossa et Abomey. Cette fonction est utilisée pour déterminer l'intervalle dans lequel pourrait se situer le seuil. Par exemple à la station de Cotonou, le seuil est compris entre 20 et $60 \mathrm{~mm}$. À la station d'Abomey, une linéarité de la fonction moyenne des excès est observée entre 15 et $45 \mathrm{~mm}$. Pour les stations de Porto-Novo et Toffo, le seuil est compris respectivement entre 10 et $35 \mathrm{~mm}$ et entre 10 et $55 \mathrm{~mm}$. La moyenne des différentes valeurs des intervalles d'estimation des seuils a permis de proposer un seuil par station d'étude. Ainsi les seuils du tableau 2 sont retenus pour les différentes stations. La plus petite valeur est obtenue à la station de Bopa avec $17.4973 \mathrm{~mm}$ tandis que la plus grande valeur est estimée à Cotonou avec $37.9033 \mathrm{~mm}$. Les séries de dépassements concernées peuvent donc être modélisées par une distribution de Pareto.

Le nombre total de dépassements de seuil varie de 522 (à Cotonou) à 1081 à Lonkly pour les stations de 1960-2012. Quant aux stations dont les données s'étendent sur 27 ans, le nombre total de dépassements 
varie de 153 à Allada et 353 à Aplahoué. Le plus petit nombre obtenu à Cotonou pourrait expliquer la part des pluies diluviennes ou de leurs cumules dans les inondations que connait la ville chaque année. A l'échelle annuelle, le nombre de jours de pluie supérieure à un seuil a relativement augmenté depuis la reprise des pluies des années 80 et 90 sur toutes les stations (figures $3 \mathrm{a}$ et $3 \mathrm{~b}$ pour les stations Toffo et Bohicon).

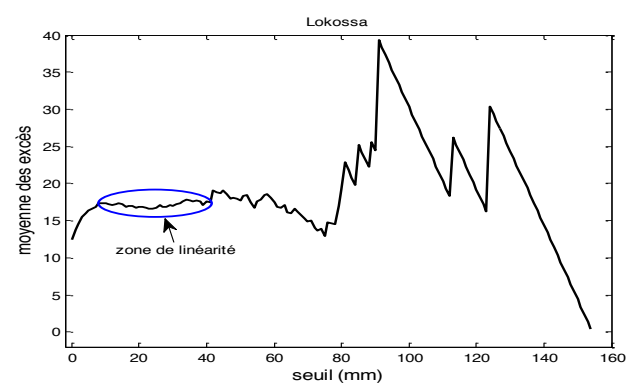

Fig.2a. Fonction moyenne des excès à Lokossa.

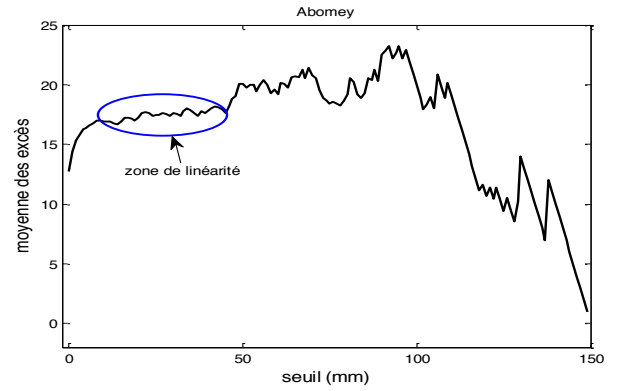

Fig.2b.Fonction moyenne des excès à Abomey.

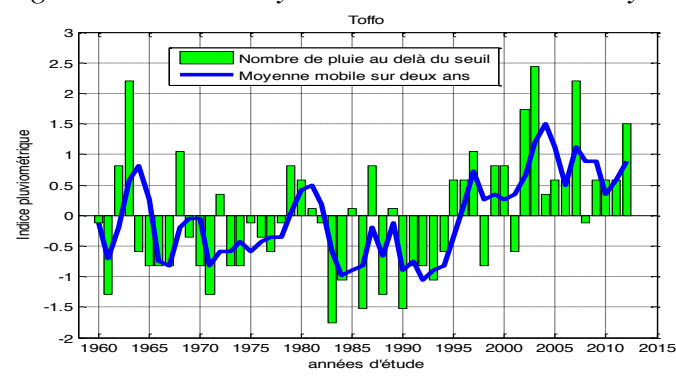

Fig.3a.Variation interannuelle du nombre de jours de pluie supérieure à $28.8501 \mathrm{~mm}$ à Toffo .

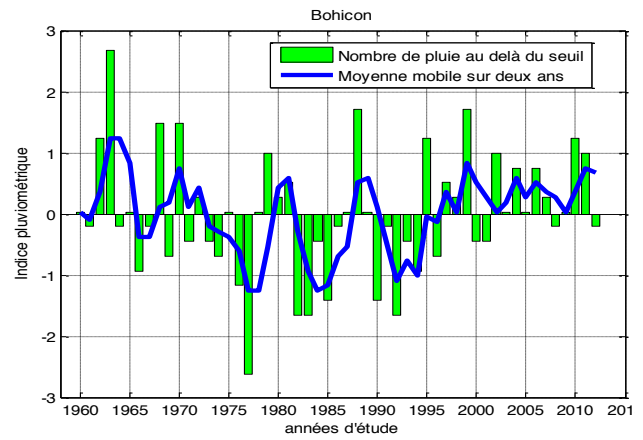

Fig.3b.Variation interannuelle du nombre de jours de pluie supérieure à $24.7631 \mathrm{~mm}$ à Bohicon .
Tableau 2 : Seuils estimés et nombres moyens annuels de dépassements des pluies journalières extrêmes.

\begin{tabular}{|l|l|l|}
\hline Stations & Seuil $(\mathrm{mm})$ & $\begin{array}{l}\text { Nombre annuel } \\
\text { Moyen de } \\
\text { dépassements }\end{array}$ \\
\hline Abomey & 28.6637 & 11 \\
\hline Adjohoun & 29.4593 & 11 \\
\hline Bohicon & 24.7631 & 14 \\
\hline Bopa & 17.4973 & 14 \\
\hline Cotonou & 37.9033 & 10 \\
\hline Grand-Popo & 26.1533 & 13 \\
\hline Kétou & 19.9214 & 18 \\
\hline Lonkly & 19.8282 & 20 \\
\hline Ouidah & 26.7354 & 13 \\
\hline Pobè & 26.7554 & 13 \\
\hline Porto-Novo & 22.3278 & 14 \\
\hline Toffo & 28.8501 & 19 \\
\hline Zagnanado & 29.4601 & 11 \\
\hline Allada & 32.4667 & 5 \\
\hline Aplahoué & 27.4348 & 12 \\
\hline Dogbo & 18.7600 & 7 \\
\hline Lokossa & 23.4727 & 7 \\
\hline
\end{tabular}

\section{II.2. Distribution statistique des pluies journalières supérieures à un seuil.}

La qualité des données constituées détermine la pertinence des résultats. Les différents tests appliqués, indiquent que les séries de dépassements obtenues sont indépendantes et identiquement distribuées. En effet, le caractère aléatoire des hauteurs de pluie constituées est mis en évidence par le test de rang de Kendall et Stuart sur les différentes stations d'étude. De plus, aucune rupture significative n'est montrée par les tests de Pettitt et de Buishand. Les faibles taux de corrélations obtenues et l'égalité de la moyenne des deux sous échantillons avec le test de Wilcoxon au seuil de défaillance de 5\% sur les différentes stations mettent en exergue l'indépendance et l'homogénéité des données dans la zone d'étude

Le tableau 3 montre les différents paramètres de la forme généralisée calculés par maximum de vraisemblance. Les paramètres de forme et d'échelle présentent une forte variabilité. Le signe significativement négatif du paramètre de forme obtenu, montre que les pluies journalières extrêmes de la zone d'étude n'appartiennent pas au domaine d'attraction de la loi de Gumbel. Ce résultat rejoint ceux de Chaouche (2001), S.A. Sisson et al. (2006), J.N. Bacro et A. Chaouche (2006), A. Muller et al. (2009) cités par Soro et al., 2016. Les plus fortes valeurs du paramètre d'échelle s'observent à Cotonou (94.6211 mm), Ouidah (75.0846 $\mathrm{mm}$ ) et Zagnanado $(76.8661 \mathrm{~mm})$ qui sont pour la plupart des stations proches de l'océan Atlantique. Ce paramètre exprime l'accroissement des pluies journalières extrêmes en fonction de la période de récurrence (Soro et al., 2016). Ce qui pourrait expliquer le risque élevé des inondations dans la zone côtière du sud Bénin. Les critères d'information Bayésien (BIC) et d'Akaike (AIC) 
sont utilisés pour dégager la loi de la famille mettant en exergue la prédominance de la forme généralisée de Pareto au détriment de la loi exponentielle dans le Bénin subéquatorial. Le tableau 4 montre les quantiles de pluie estimés à Porto-Novo et Grand-Popo. Les quantiles de pluie estimés sur les différentes stations augmentent avec les années de retour comme sur l'ensemble de la Côte d'Ivoire (Soro et al., 2016).

Tableau 3: Les paramètres de forme, d'échelle et de position calculés par maximum de vraisemblance.

\begin{tabular}{|l|l|l|l|}
\hline \multirow{2}{*}{ Stations } & \multicolumn{3}{|c|}{ Paramètres } \\
\cline { 2 - 4 } & $\begin{array}{l}\text { Forme } \\
(\mathbf{k})\end{array}$ & $\begin{array}{l}\text { Echelle } \\
(\boldsymbol{\alpha}) \\
\mathbf{m m}\end{array}$ & $\begin{array}{l}\text { Position } \\
\text { (u) en mm }\end{array}$ \\
\hline Abomey & -0.4614 & 70.3436 & 28.6637 \\
\hline Adjohoun & -0.3620 & 69.1291 & 29.4593 \\
\hline Bohicon & -0.5304 & 68.8087 & 24.7631 \\
\hline Bopa & -0.4693 & 61.1771 & 17.4973 \\
\hline Cotonou & -0.4734 & 94.6211 & 37.9033 \\
\hline Grand-Popo & -0.3193 & 70.3235 & 26.1533 \\
\hline Kétou & -0.3784 & 60.0282 & 19.9214 \\
\hline Lonkly & -0.4222 & 62.6966 & 19.8282 \\
\hline Ouidah & -0.3430 & 75.0846 & 26.7354 \\
\hline Pobè & -0.4909 & 69.2816 & 26.7554 \\
\hline Porto-Novo & -0.3239 & 69.3378 & 22.3278 \\
\hline Toffo & -0.2655 & 61.7907 & 28.8501 \\
\hline Zagnanado & -0.5816 & 76.8661 & 29.4601 \\
\hline Allada & -1.2418 & 84.4452 & 32.4667 \\
\hline Aplahoué & -1.3911 & 70.6657 & 27.4348 \\
\hline Dogbo & -1.0931 & 65.0413 & 18.7600 \\
\hline Lokossa & -1.2740 & 100.6486 & 23.4727 \\
\hline
\end{tabular}

Tableau 4 : Quantiles de pluies (en mm) obtenues avec la forme généralisée de la loi de Pareto à Porto-Novo et Grand-Popo.

\begin{tabular}{|l|l|l|l|l|}
\hline \multirow{2}{*}{ Stations } & \multicolumn{4}{|l|}{ Période de retour (ans) } \\
\cline { 2 - 5 } & $\mathbf{6}$ & $\mathbf{1 0}$ & $\mathbf{3 0}$ & $\mathbf{1 0 0}$ \\
\hline $\begin{array}{l}\text { Porto- } \\
\text { Novo }\end{array}$ & 190.7305 & 259.5497 & 452.4235 & 759.6473 \\
\hline $\begin{array}{l}\text { Grand- } \\
\text { Popo }\end{array}$ & 196.1962 & 265.3515 & 458.4448 & 764.4198 \\
\hline
\end{tabular}

\section{CONCLUSION}

L'analyse des pluies du sud Bénin à partir d'un seuil donné a permis d'identifier plusieurs valeurs extrêmes par année d'étude. Ces valeurs extrêmes ont relativement augmenté sur les différentes stations d'étude avec la reprise de la pluviométrie depuis la fin des années 80 ou le début des années 90. Le paramètre de forme est significativement négatif sur les différentes stations d'étude. La forme généralisée des lois de Pareto estime mieux les séries de pluies journalières extrêmes sur la période de 1960 à 2012. La plupart des valeurs élevées du paramètre d'échelle est obtenue sur des stations proches de l'océan Atlantique, montrant ainsi le risque élevé des inondations sur la côte béninoise.
Dans le contexte actuel de changement climatique, la grande inconnue qui demeure est l'évolution future de l'intensité et de la fréquence de ces évènements pluvieux surtout que les travaux du GIEC évoquent l'intensification des paramètres climatiques dans le futur dans plusieurs régions du monde. L'élaboration et la mise en œuvre des schémas d'expansion de nos villes doivent prendre en compte le risque climatique par nos décideurs à travers des mesures idoines de prévention et d'adaptation. Il devient donc impérieux de développer des outils de prévision et de simulation des crues.

\section{REFERENCES}

Acero F.J., Gracia J.A. et Gallego M.C. (2011) - Peaksover-threshold study of trends in extreme rainfall over the Iberian Peninsula. Journal of Climate, vol. 24, $\mathrm{n}^{\circ}$ 4, p. 1089-1105.

Adewi E., K. M. S. Badameli et V. Dubreuil (2010), Evolution des saisons des pluies potentiellement utiles au Togo de 1950 à 2000, Climatologie, 7, 92-94.

Akaike, H. (1974). A new look at statistical model identification. IEEE Trans. Auto. Control, 19 :716722 .

Alain I. AGUE et Abel AFOUDA, 2015. Analyse fréquentielle et nouvelle cartographie des maxima annuels de pluies journalières au Bénin. International Formulae Group. All rights reserved. DOI: http://dx.doi.org/10.4314/ijbcs.v9i1.12

Anadia Niger, 2014. Manuel de la base de données des inondations.

Brahim Habibi et al. 2012. Analyse fréquentielle des pluies journalières maximales Cas du Bassin ChottChergui. Nature \& Technologie, 41-48.

Beirlant, J., Goegebeur, Y., Teugels, J. \& Segers, J. (2004). Statistics of Extremes: Theory and Applications, Wiley, England.

Beirlant, J. \& Guillou, A. (2001). Pareto index estimation under moderate right censoring. Scand. Actuar. J., 2, 111-125.

Coles, S. (2001). An Introduction to Statistical Modeling of Extreme Values. Bristol, UK: Springer, 221 p.

Daniel Gellens (2002). Combining regional approach and data extension procedure for assessing GEV distribution of extreme precipitation in Belgium. Journal of Hydrology 268 (2002) 113-126.

Gneneyougo Émile Soro, Amidou Dao, Vamoryba Fadika, Tié Albert Goula BI et Bernard Srohorou, 2016. Estimation des pluies journalières extrêmes supérieures à un seuil en climat tropical : cas de la Côte d'Ivoire. Physio-Géo - Géographie Physique et Environnement, 2016, volume X.

Goubanova Katerina, Une étude des événements climatiques extrêmes sur l'Europe et le bassin 
Méditerranéen et de leur évolution future. Thèse de Doctorat, UNIVERSITÉ de PARIS 6, 119P.

Hangnon, H., de Longueville, F., Ozer, P., 2015. Précipitations "extrêmes" et inondations à Ouagadougou; quand le développement urbain est mal maîtrisé. Presented at the Actes du 28e Colloque.

Hountondji, Y., De Longueville, F., Ozer, P., 2011. Trends in extreme rainfall events in Benin (West Africa), 1960-2000. Presented at the Proceedings of the 1st International Conference on Energy, Environment and Climate Change. hdl.handle.net/2268/96112.

Inondation au Bénin - Rapport d'évaluation des Besoins Post Catastrophe. Préparé par le Gouvernement de la République du Bénin avec l'appui de la Banque Mondiale et du Système des Nations Unies. Rapport Final Avril, 2011.

Koutsoyiannis D, Baloutsos G. 2000. Analysis of a long record of annual maximum rainfall in Athens, Greece and design rainfall inferences. Natural Hazards (Dordrecht), 22(1): 29-48.

Koutsoyiannis D. 2004a. Statistics of estimation of extreme rainfall: I. Theoretical of investigation. Journal desSciences Hydrologiques, 49(4) : 575-590.

Lawin A. E., Analyse climatologique et statistique du régime pluviométrique de la haute vallée de l'Ouémé à partir des données pluviométriques Amma-CATCH Bénin, thèse de Doctorat, Institut Nationale Polytechnique de Grenoble et l'Université d'AbomeyCalavi.

Neppel L., Bouvier C., Vinet F., Desbordes M., 2003. Sur l'origine de l'augmentation apparente des inondations en région méditerranéenne, Revue des Sciences de l'Eau, 16, 475-493.

Ozer, P., 2012. Les risques naturels. Articuler risques, planification d'urgence et gestion de crise. Editions de Boeck, 135p.

Ozer, P., Bodart, C., Tychon, B., 2005. Analyse climatique de la région de Gouré, Niger oriental : récentes modifications et impacts environnementaux. CyberGeo : European Journal of Geography. cybergeo.revues.org/3338. Consulté le 23/09/2016.

Panthou, G., 2013. Analyse des extrêmes pluviométriques en Afrique de l'Ouest et de leur évolution au cours des 60 dernières années. Thèse de Doctorat, Université de Grenoble, France, 270p.

Pickands J. (1975) - Statistical Inference Using Extreme Order Statistics. Annals of Statis tics, vol. 3, $\mathrm{n}^{\circ}$ 1, $\mathrm{p}$. 119-131.

Sahani, M., Moeyersons, J., Vandecasteele, I., Trefois, P., Ozer, P., 2012. Evolution des caractéristiques pluviométriques dans la zone urbaine de Butembo (RDC) de 1957 à 2010. Geo-Eco-Trop : Revue Internationale de Géologie, de Géographie et d'Ecologie Tropicales 36, 121-136.
H. Saidi, M. Ciampittiello, C. Dresti, and G. Ghiglieri. Observed variability and trends in extreme rainfall indices and Peaks-Over-Threshold series. Hydrol. Earth Syst. Sci. Discuss., 10, 6049-6079, 2013

SENE, S. \& OZER, P., 2002. Evolution pluviométrique et relation inondations-événements pluvieux au Sénégal. Bulletin de la société géographique de Liège, 42:27-43.

Tschakert, P., 2007. Views from the vulnérable: Understanding climatic and other stressors in the Sahel". Global Environmental Change. Vol. 17. n ${ }^{\circ}: 3-$ 4, p. 381-396.

WMO, 2009. Guidelines on analysis of extremes in a changing climate in support of informed decisions for adaptation. World Meteorological Organization, Genève, $52 \mathrm{p}$. 